\title{
Models of Pedestrian Evacuation based on Cellular Automata
}

\author{
M.J. Mrowinski ${ }^{a}$, T.M. Gradowski $^{a}$, R.A. Kosinski ${ }^{a, b}$ \\ ${ }^{a}$ Faculty of Physics, Warsaw University of Technology, Koszykowa 75, 00-662 Warszawa, Poland \\ ${ }^{b}$ Central Institute of Labor Protection - National Research Institute, Czerniakowska 16, 00-701 Warszawa, Poland \\ We study two models of pedestrian evacuation based on cellular automata and intelligent agents. We use the \\ static floor field approach and augment it with additional transition rules (random movement and preservation \\ of personal space) in order to model various aspects of human behaviour. Through numerical simulations we \\ investigate pedestrian evacuation from rooms with various geometries (e.g. empty room, classroom). Using \\ heatmaps (density diagrams of pedestrians), average evacuation times, velocity and other parameters we study \\ the effectiveness of evacuation and potential dangers pedestrians may encounter.
}

PACS: 89.40.-a, 45.70.Vn, 05.50.+q, 05.70.Fh

\section{Introduction}

The problem of pedestrian evacuation has attracted a lot of attention in the last few years [1]. Scientists from many different fields used various mathematical and physical approaches to model and study this phenomenon. Such studies are very important and may lead to an increase in safety and better understanding of the dangers pedestrians face during evacuation.

Existing models of pedestrian evacuation are based on either the Langevin equation [2-6] or cellular automata [7-13] (for an introduction to cellular automata see $[14,15])$. In the Langevin equation approach both time and space are continuous. Pedestrians are treated like particles interacting with the surrounding world through so called social forces. Models based on cellular automata are intrinsically discrete and usually employ various kinds of static and dynamic floor fields to govern the motion of pedestrians.

In [16] we introduced two simple models of pedestrian motion based on cellular automata. Our goal was to simplify the usual cellular automata approach (which often involves complicated rules) and create a modular environment where simple rules can be connected using easily understandable parameters to form complex systems with rich behaviour. Both model were used to simulate evacuation on staircase-like geometries. In this work we present some general properties of our two models in their most basic form using two most commonly studied geometries - empty room and classroom (Fig. 1).

\section{Model}

We assume that space is discrete, much like in a twodimensional cellular automata, and divided into square cells of equal size. There are three kinds of cells: floor cells (without any additional properties), exit cells

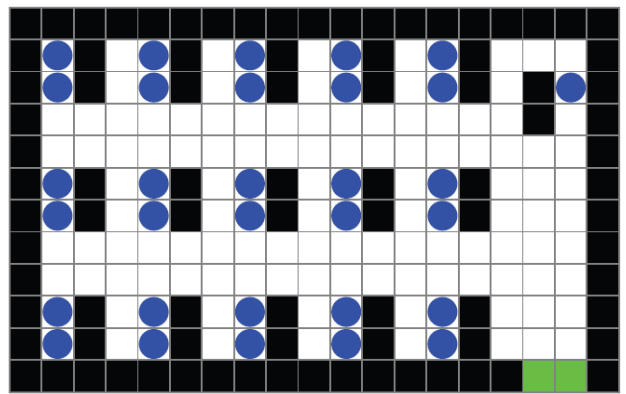

Fig. 1. Classroom with 30 students and 1 teacher.

(pedestrians at these cells are removed from the system) and wall cells (obstacles that cannot be occupied). Each cell can be occupied by only one pedestrian. Time is also discrete, with pedestrians moving asynchronously in a random order (shuffle update). Both models presented in this paper are build on top of a simple static floor field introduced in [9]. In this approach, each cell is assigned a value according to the following, iterative algorithm:

1. Exit cells are assigned value 1.

2. During subsequent iterations each cell that was assigned a value $(v)$ in the previous iteration tries to assign a value to all its neighbours: $v+1$ to the horizontal and vertical neighbours, $v+1.5$ to the diagonal ones. If this procedure leads to a conflict (when two cells try to assign a value to the same neighbouring cell) then the lowest floor field value is used.

3. Wall cells, to make them inaccessible, are assigned the highest possible floor field value.

The resulting floor field values can be interpreted as the distance from a given cell to exit cells and are very similar 
to the Manhattan metric. In this model, however, motion in the vertical direction is also possible. The floor field defined above can be used, on its own, to simulate pedestrian movement. In our work we use it as a basis and augment it, in a modular fashion, with additional, simple rules. Such an approach allows to easily decompose various aspect of pedestrian motion into smaller, logical elements and study their influence on the overall dynamics of pedestrians.

The first variation of the above model we want to present is called floor field model with random movement (FFRM for short). FFRM is similar to biased random walkers introduced by Nagatani $[7,8]$. In FFRM pedestrians move:

1. with probability $(1-\beta)$ to the neighbouring cell with the lowest floor field value (as long as this value is lower than the floor field value of the cell currently occupied by the pedestrian),

2. with probability $\beta$ to a random neighbouring cell.

When $\beta=0$, pedestrians move according to the pure static floor field and simply follow the shortest path to the exit. With $\beta=1$, pedestrians behave like random walkers - with equal probability they move in any available direction even if it takes them away from the exit. For intermediate values of $\beta$, these two behaviour intertwine. It should be noted that this model is a two dimensional equivalent of a one dimensional ASEP (asymmetric simple exclusion process). ASEP, a simple model of particle transport, have successfully been used to model car traffic.

Our second variation of the static floor field model is called floor field with pressure (FFP). When moving according to FFP rules, pedestrians try to preserve some personal space, that is to minimise their number of neighbours. In order to do that, they move:

1. to the neighbouring cell with the lowest floor field value (as long as this value is lower than the floor field value of the cell currently occupied by the pedestrian) with probability 1 when there are no other pedestrians in the neighbouring cells, or with probability $(1-\beta)$ when there are other pedestrians,

2. to a neighbouring cell surrounded by the smallest number of pedestrians with probability $\beta$ when there are other pedestrians in the neighbourhood.

It means that for $\beta=0$, much like in FFRM, pedestrians move according to the pure static floor field model. When $\beta=1$, they still follow the shortest path to the exit as long as there are no other pedestrians in their vicinity. However, if there is at least one neighbour in their neighbourhood, then they will always try to move away.

The biggest difference between FFRM and FFP lies in the way both rules are applied for intermediate values of $\beta$. In case of FFRM, pedestrians always move either randomly or in the direction of the exit. In FFP, however, their behaviour is highly dependent on the number of neighbours. Even when $\beta=1$, it is possible for pedestrians moving according to FFP, as long as they do not encounter any other pedestrians, to never stray from the shortest path.

In FFRM $\beta$ can be regarded as a measure of panic or irrationality in the behaviour of pedestrians. Low values of $\beta$ correspond to low levels of panic/irrationality, high values correspond to high levels. Using this interpretation we can choose $\beta$ appropriate for the simulated environment and situation. For example, when there is no immediate danger or the evacuation process is efficient due to training or professional execution, pedestrians are more likely to make rational choices. However, parameter $\beta$ in FFP plays a slightly different role (it is a measure of a certain psychological inclination) and the proper choice of $\beta$ would require a more detailed empirical study. One could expect that people, when facing danger, would be willing to sacrifice certain level of comfort by reducing their personal space.

\section{Measured quantities}

Before we begin to present our results it is important to specify exactly what quantities we measure during simulations. One of the most important and widely used is average evacuation time. Average evacuation time is defined as the number of time steps a pedestrian spends in the system before exiting through one of the exits, averaged over all pedestrians and then averaged over multiple realisations of the system. Average evacuation time is a measure of the efficiency of evacuation and, generally speaking, the lower the better.

Using average evacuation time we constructed another possible measure - average relative evacuation time. It is defined as the number of time steps a pedestrian spends in the system divided by the average number of time steps a pedestrian starting at the same cell would spend in the system if they were the only pedestrian in the system (averaged over all pedestrians and multiple realisations). To put it simply, in order to calculate average relative evacuation time we place a pedestrian in a cell (we do it for all cells, one cell at a time) and calculate the average evacuation time for this particular cell. Once it is done, during the actual simulation, pedestrians remember the average evacuation time associated with their starting cells and when they leave the system, their evacuation time is divided by this value. Average relative evacuation time allows us to see how other pedestrians in the system affect the evacuation process.

In most papers systems like ours are visualised by drawing simple snapshots at some time step $t$. We use a different approach which, in our opinion, contains much more information. Instead of drawing snapshots, we construct density diagrams - roughly speaking, images combining information from multiple realisations of the system. In order to create a density diagram we have to 
define an occupancy function

$$
c_{i j}^{(k)}(t)=\left\{\begin{array}{l}
1 \text { if there is a pedestrian at cell } i j \\
\text { during time step } t \\
0 \begin{array}{l}
\text { if cell } i j \text { is unoccupied during } \\
\text { time step } t
\end{array}
\end{array}\right.
$$

which tells us whether cell $i j$ was occupied by a pedestrian at time step $t$ in the $k$-th realisation of the system. Using this function we can calculate the probability that cell $i j$ will be occupied at time step $t$ by averaging $c_{i j}^{(k)}(t)$ over multiple realisations

$$
d_{i j}(t)=\frac{1}{N} \sum_{k=1}^{N} c_{i j}^{(k)}(t)
$$

The last step in creating a density diagram is to map $d_{i j}(t)$ into some colour scale (a heat-map). It should be noted that this definition varies slightly from the one presented in [16]. Due to the nature of the system studied in that paper the average was taken over time rather than realisations. The definition introduced above is a more general one.

\section{Results}

In this section we present some general properties of our models using simple, frequently studied geometries - empty room and classroom (see [16] for a more complicated case). Empty room consists of a rectangular grid with $L^{2}$ cells (in all simulations presented in this paper $L=25$ which means pedestrians can occupy $(L-2)^{2}=526$ cells $)$. Sides of the rectangle are set to wall cells except for a single side where $l$ cells in the middle are set to exit cells (doors). At the beginning of each simulation pedestrians are randomly distributed in the room.

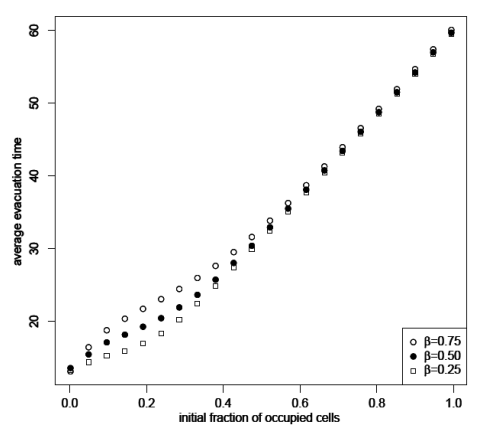

Fig. 2. Empty room (FFP): average evacuation time as a function of initial fraction of occupied cells for different values of $\beta$.

For FFP, both average evacuation time and average relative evacuation time increase with initial fraction of occupied cells (Figs. 2, 3). For low values of initial fraction of occupied cells $\beta$ has got a high influence on evacuation time - when pedestrians prioritise the preservation of personal space it takes them longer to reach the exit. However, as the number of pedestrians in the system increases, the impact of $\beta$ on the dynamics grows

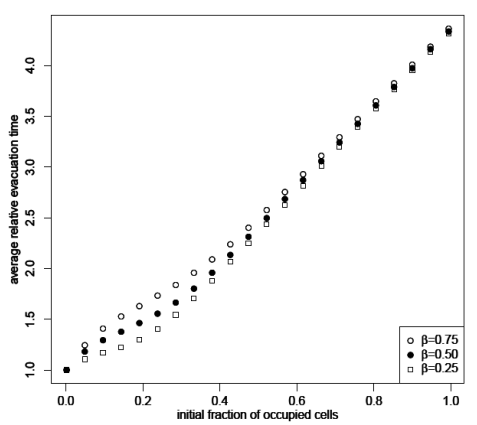

Fig. 3. Empty room (FFP): average relative evacuation time as a function of initial fraction of occupied cells for different values of $\beta$.

smaller and the difference between evacuation times becomes negligible. Such a behaviour can be easily explained by the fact that for high values of initial fraction of occupied cells pedestrians quickly form a big cluster (spanning nearly the entire room at the beginning) around the exit. Pedestrians next to the exit are pushed out from the system, pedestrians in the middle are either stuck or pushed towards the exit and pedestrians at the rim of the cluster (the ones influenced by $\beta$ the most) try to separate from it by moving away, but they still stay in its vicinity. The cluster shrinks and eventually disappears. It follows that the longer it takes for the cluster to form the more important are the interactions between individual pedestrians in the time preceding its formation (when many pedestrians interact).

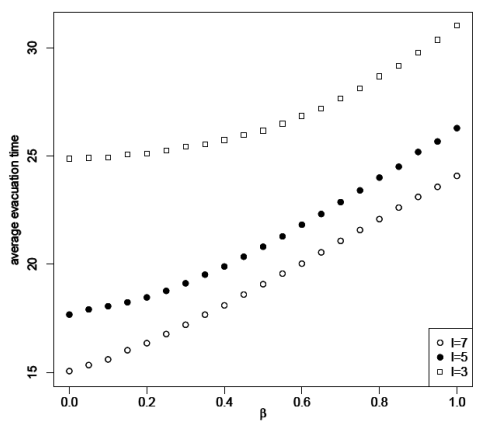

Fig. 4. Empty room (FFP): average evacuation time as a function of $\beta$ for initial fraction of occupied cells $=$ $1 / 4$ and various door lengths.

When initial fraction of occupied cells is set, average evacuation time and average relative evacuation time in FFP increase with $\beta$ (Figs. 4, 5) and for high values of $\beta$ their dependency is linear. When we increase the width of the exit pedestrians are able to leave the system faster. It should be noted that average evacuation time and average relative evacuation time do not change linearly with the width of the exit.

Contrary to what we can observe in FFP, average evacuation time in FFRM depends highly on $\beta$ (Fig. 6) for all 


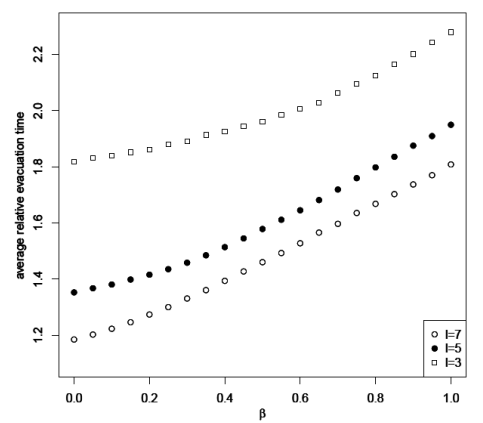

Fig. 5. Empty room (FFP): average relative evacuation time as a function of $\beta$ for initial fraction of occupied cells $=1 / 4$ and various door lengths.

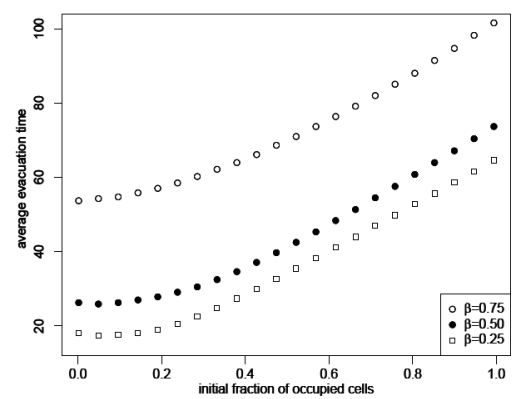

Fig. 6. Empty room (FFRM): average evacuation time as a function of initial fraction of occupied cells for different values of $\beta$.

values of initial fraction of occupied cells. When initial fraction of occupied cells is low, average relative evacuation time is almost independent of $\beta$ (Fig. 7). The reason for that is simple - unlike FFP, interactions in FFRM are implicit (resulting from the fact that two pedestrians cannot occupy the same cell). With only a few pedestrians present in the system, relative to the total number of available cells, such interactions are negligible. For high values of $\beta$ average relative evacuation time increases with initial fraction of occupied pedestrians linearly.

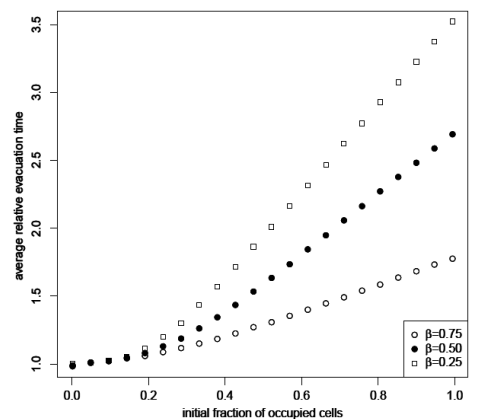

Fig. 7. Empty room (FFRM): average relative evacuation time as a function of initial fraction of occupied cells for different values of $\beta$.

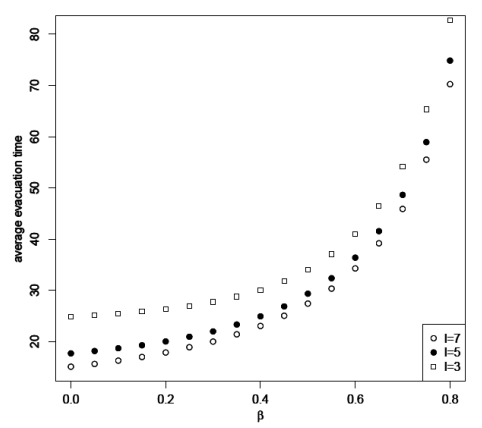

Fig. 8. Empty room (FFRM): average evacuation time as a function of $\beta$ for initial fraction of occupied cells $=$ $1 / 4$ and various door lengths.

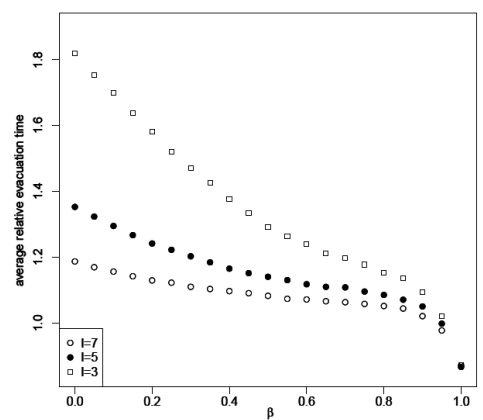

Fig. 9. Empty room (FFRM): average relative evacuation time as a function of $\beta$ for initial fraction of occupied cells $=1 / 4$ and various door lengths.

When initial fraction of occupied cells is set, average evacuation time in FFRM increases with $\beta$ (Fig. 8). However, the dependency between average relative evacuation time and $\beta$ in FFRM is much different than its FFP counterpart. Average relative evacuation time decreases with increasing $\beta$ and for very high values of $\beta$ it is lesser than one. It means that pedestrians in the system actually help each other to reach the exit. A similar, though more interesting behaviour (with the same underlying cause) can be observed in staircases [16].

Figure 10 shows density diagrams for empty room. The influence of $\beta$ is clearly visible - for both FFP and FFRM the cluster forming around the exit is more diluted. While evacuation time is inevitably greater than in pure floor field model, the decrease in pedestrian density increases the overall safety of the evacuation process.

The geometry of a typical classroom is depicted in Fig. 1. In the examples presented in this paper we assume there are 30 students in the classroom, placed at the beginning of the simulation in front of their desks. Desks are arranged in three columns. The teacher stands next to their desk, near the blackboard. The exit, 2 cells wide, is located on the same wall as the blackboard.

The results of simulations obtained for classroom geometry are similar to the ones we observed for an empty room. When FFP rules are used, average evacuation 

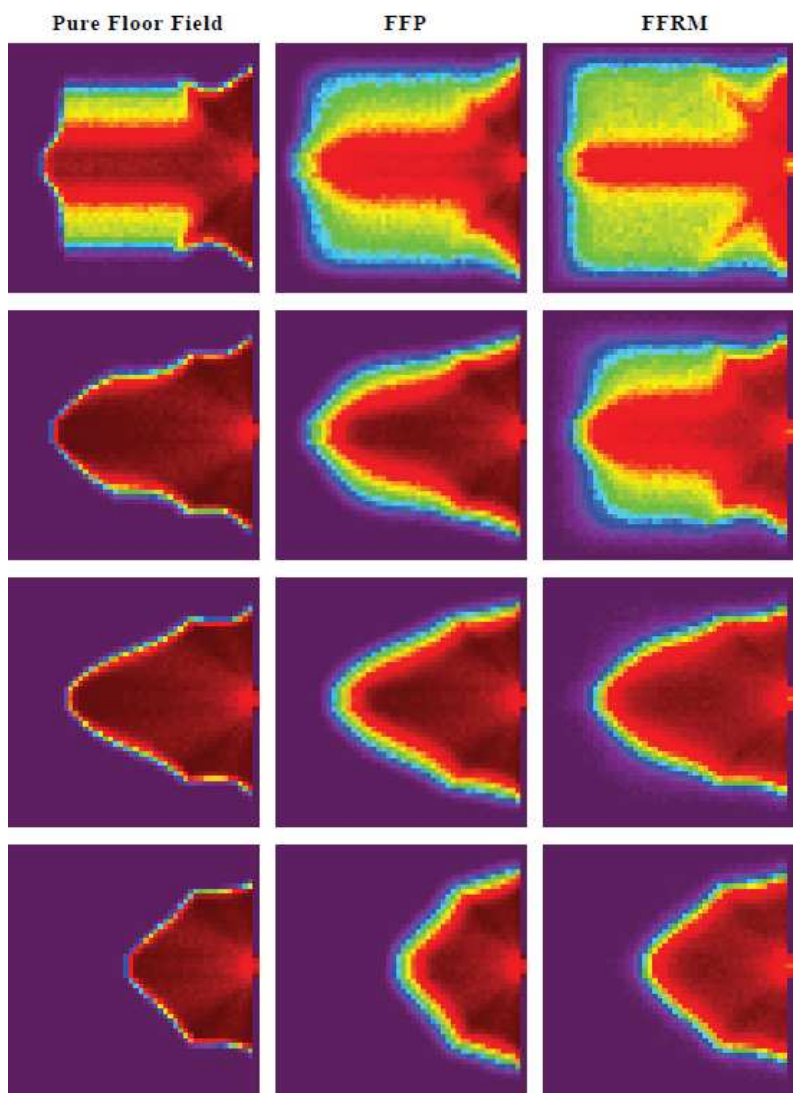

Fig. 10. Empty room: density diagram for $\beta=0.5$ at three different time steps (time increases from top to bottom). The warmer the colour the higher the density.

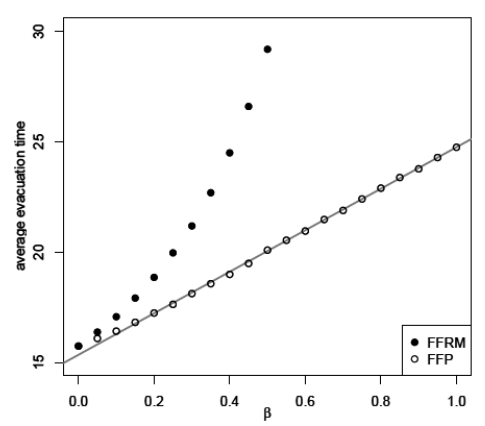

Fig. 11. Classroom: average evacuation time as a function of $\beta$.

time, after initial nonlinear period for low $\beta$, changes linearly with $\beta$ (Fig. 11). This dependency is always nonlinear for FFRM. Average relative evacuation time, as in an empty room, exhibit a more interesting behaviour (Fig. 12). It increases with $\beta$ for FFP, but decreases for FFRM. For high values of $\beta$ average relative evacuation time is lesser than one - it means that for a highly random behaviour pedestrians help each other to reach the exit.

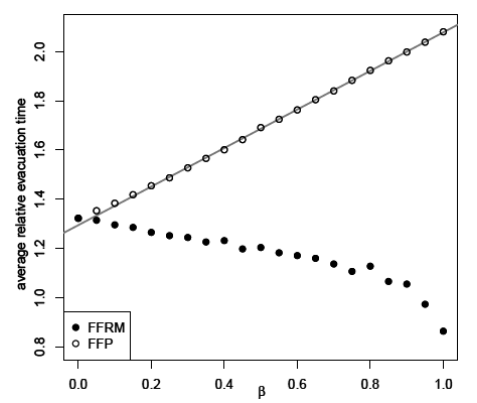

Fig. 12. Classroom: average relative evacuation time as a function of $\beta$.

Density diagrams (Fig. 13) also confirm, even more vividly, that density of pedestrians is lower for FFP and FFRM than in pure floor field model. Clusters of pedestrians that inevitably form near the exit are more diluted, which means evacuating pedestrians are less likely to, for example, sustain injuries. It is interesting that seemingly irrational behaviour can increase the safety of pedestrians.

\section{Conclusions}

The two models presented in this paper, despite their simplicity, seem to mimic various aspects of human behaviour. The models are general and can be used for any geometry (for example, with a clever manipulation of floor field values, it should be possible to combine systems like rooms and staircases into one, big system). Their modular nature allows for an easy composition of complex systems with multiple layers of interacting rules.

The average relative evacuation time and density diagrams are useful tools that provide interesting information about the dynamics. These measures can be used in any cellular automata based and (without much additional effort) continuous models. Density diagrams seem to be a good alternative for simple snapshots and are helpful in localising threats that would otherwise be overlooked.

Simulations performed for an empty room and classroom confirm our findings presented in [16] for staircaselike geometries. While it is tempting to associate the efficiency of evacuation with average evacuation time, often other factors are important and cannot be overlooked. Following the shortest path to the exit is not always the best option. While irrational behaviour increases the evacuation time, it can lead to an overall increase in safety.

\section{Acknowledgements}

This work was supported by the European Cooperation in the field of Scientific and Technical Research COST Action MP0801 "Physics of competition and conflicts". 


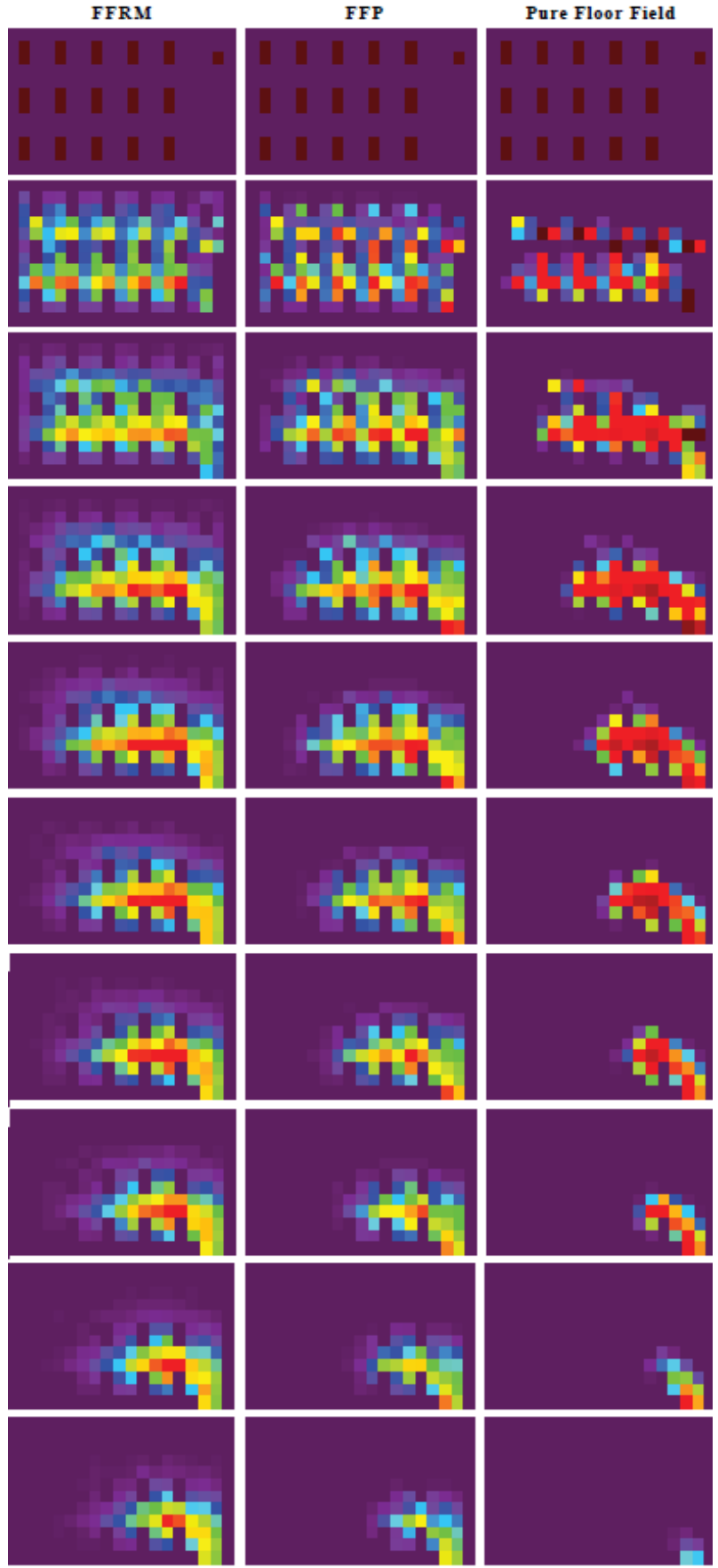

Fig. 13. Classroom: density diagram for $\beta=0.5$ at different time steps (time increases from top to bottom).

\section{References}

[1] A. Schadschneider, W. Klingsch, H. Klüpfel, T. Kretz, C. Rogsch, A. Seyfried, Evacuation Dynamics: Empirical Results, Modeling and Applications in: Encyclopedia of Complexity and System Science, Ed. R.A. Meyers, Springer, Berlin 2009, p. 3142.

[2] D. Helbing, P. Molnar, Phys. Rev. E 51, 4282 (1995).

[3] D. Helbing, I. Farkas, T. Vicsek, Nature 407, 487 (2000).

[4] D. Helbing, Quantitative Sociodynamics: Stochastic Methods and Models of Social Interaction Processes, Kluwer Academic, Dordrecht 1995.

[5] D.R. Parisi, C.O. Dorso, Physica A 354, 606 (2005).

[6] R.A. Kosinski, A. Grabowski, Acta Phys. Pol. B Proc. Suppl. 3, 1001 (2010).

[7] Y. Tajima, K. Takimoto, T. Nagatani, Physica A 294, 257 (2001).

[8] Y. Tajima, T. Nagatani, Physica A 303, 239 (2002).

[9] A. Varas, M.D. Cornejo, D. Mainemer, B. Toledo, J. Rogan, V. Munoz, J.A. Valdivia, Physica A 382, 631 (2007).

[10] H. Huang, R. Guo, Phys. Rev. E 78, 021131 (2008).

[11] C. Burstedde, K. Klauck, A. Schadschneider, J. Zittartz, Physica A 295, 507 (2001).

[12] W. Song, X. Xu, B. Wang, S. Ni, Physica A 363, 492 (2006).

[13] J. Zhang, W. Song, X. Xu, Physica A 387, 5901 (2008).

[14] A. Ilachinski, Cellular Automata: A Discrete Universe, World Scientific Publishing, Singapore 2001.

[15] S. Wolfram, Rev. Mod. Phys. 55, 601 (1983).

[16] M.J. Mrowinski, R.A. Kosinski, Int. J. Mod. Phys. C 22, 71 (2011) 